\title{
Evaluation of Multiple Tracer Methods to Estimate Low Groundwater Flow Velocities
}

Paul W. Reimus ${ }^{1}$ and Bill W. Arnold ${ }^{2,3}$

${ }^{1}$ Los Alamos National Laboratory, Los Alamos, NM, preimus@lanl.gov, corresponding author

${ }^{2}$ Sandia National Laboratories, Albuquerque, NM

${ }^{3}$ Current Affiliation: Arnold Hydrogeology Consulting, 4756 SW Lowell Ct., Portland, OR 97221, bwarnol@gmail.com

\begin{abstract}
Four different tracer methods were used to estimate groundwater flow velocity at a multiplewell site in the saturated alluvium south of Yucca Mountain, Nevada: (1) two single-well tracer tests with different rest or "shut-in" periods, (2) a cross-hole tracer test with an extended flow interruption, (3) a comparison of two tracer decay curves in an injection borehole with and without pumping of a downgradient well, and (4) a natural-gradient tracer test. Such tracer methods are potentially very useful for estimating groundwater velocities when hydraulic gradients are flat (and hence uncertain) and also when water level and hydraulic conductivity data are sparse, both of which were the case at this test location. The purpose of the study was to evaluate the first three methods for their ability to provide reasonable estimates of relatively low groundwater flow velocities in such low-hydraulic-gradient environments. The natural-gradient method is generally considered to be the most robust and direct method, so it was used to provide a "ground truth" velocity estimate. However, this method usually requires several wells, so it is often not practical in systems with large depths to groundwater and correspondingly high well installation costs. The fact that a successful natural gradient test was conducted at the test location offered a unique opportunity to compare the flow velocity estimates obtained by the
\end{abstract}


more easily deployed and lower risk methods with the ground-truth natural-gradient method. The groundwater flow velocity estimates from the four methods agreed very well with each other, suggesting that the first three methods all provided reasonably good estimates of groundwater flow velocity at the site. The advantages and disadvantages of the different methods, as well as some of the uncertainties associated with them are discussed.

\section{KEYWORDS}

Tracer, Tracer Test, Groundwater Velocity, Specific Discharge

\section{INTRODUCTION}

Estimates of risks associated with groundwater contamination are typically very sensitive to groundwater flow velocity estimates at the site of interest. As an example, saturated zone model sensitivity studies conducted in support of the license application for the proposed high-level nuclear waste repository at Yucca Mountain, Nevada indicated that the most important parameter affecting predicted radionuclide concentrations and doses at the compliance boundary was the saturated zone flow velocity (Arnold et al., 2008). The saturated alluvium south of Yucca Mountain represented the final barrier to radionuclide migration from the proposed repository to the accessible environment (defined by a regulatory compliance boundary approximately $18 \mathrm{~km}$ from Yucca Mountain). Consequently, the flow and transport properties of this alluvium were the subject of much scientific investigation, and considerable effort was put into obtaining estimates of the ambient flow velocity in the alluvium. Estimates based on hydraulic gradients from water level measurements and hydraulic conductivities from aquifer pump tests were considered quite uncertain because of the shallowness of the horizontal gradient, a strong vertical 
gradient (making water levels a strong function of screen depth), and sparseness of wells in the alluvium. Thus, there was an incentive to obtain groundwater flow velocity estimates using methods other than the conventional approach of multiplying hydraulic conductivity and gradient.

In 2004 and 2005, two single-well injection-withdrawal tracer tests and two cross-hole forced-gradient tracer tests were conducted in the saturated alluvium at Nye County Early Warning Drilling Program Site 22 (NC-EWDP Site 22). The results and interpretations of these tests were previously reported by Reimus et al. (2006), with details of the method used to estimate flow velocity from the single-well tests described in Reimus et al. (2003a). However, these authors did not discuss the planned flow interruption during one of the cross-hole tests or the ambient flow velocity estimates that could be obtained from analyzing the tracer responses to this flow interruption. From August 2006 to October 2008, a natural gradient tracer test was conducted at Site 22. The results and interpretations of this test have not previously been reported other than in Yucca Mountain Project reports that have had limited distribution.

NC-EWDP Site 22 is located about $14 \mathrm{~km}$ south of Yucca Mountain with one large-diameter $\left(6.75^{\prime \prime}\right)$ well that can be pumped at a relatively high rate (22S) and three 2-inch piezometers (22PA, 22PB, and 22PC) that can be used as observation wells in hydraulic testing or injection wells in cross-hole tracer testing. The site location and layout are shown in Figure 1. The site is situated along a projected flow pathway from the proposed repository, so it is a good location for assessing flow and transport in the saturated alluvium. A summary of the well completions and site geology is provided in Figure 2. All of the tracer tests discussed in this paper were conducted in the second screened interval from the top of the wells, which was more transmissive than the uppermost interval and thus considered to be the more likely radionuclide 
transport pathway and also the pathway that would result in the most rapid (pessimistic) travel times. Note that the local horizontal hydraulic gradient at the site was indeterminate because the water levels in the different wells of Figure 1 were essentially indistinguishable in screened intervals completed at the same depth.

The objective of this paper is to provide the reader with some perspective on the advantages and disadvantages of each of the three tracer-based methods for estimating natural flow velocities, with the natural gradient test result serving as a "ground truth". While natural gradient tests certainly provide the best possible information, they can be expensive and risky in deep systems that require costly well installations. They typically require many wells to ensure success because slight deviations in true flow direction from anticipated flow direction or inadvertent installation of wells into low permeability subdomains can result in tracers completely missing downgradient wells. In this regard, it was considered quite fortuitous that a successful natural-gradient test was conducted for this study with only a single downgradient well. This fortunate result provided the opportunity to evaluate the ability of the other methods to provide reasonable estimates of natural flow velocity. In the interest of space, the experimental aspects of the methods are only briefly summarized herein; the reader is referred to the citations for experimental details.

\section{METHODS}

Four different tracer methods were used to estimate ambient flow velocity in the saturated alluvium at Site 22. These methods are described briefly in this section, with references provided for additional detail. The second and third methods are described in the greatest detail because the first method has been previously described (Reimus et al., 2003a), and the fourth 
method (borehole dilution) is a standard, well-accepted method. We note that in all the methods, any unnatural flow conditions induced by injection or pumping either contributed very little to overall tracer migration, or, in the case of the multiple single-well tracer tests, their effects were removed from the test interpretations by ensuring that the exact same flow conditions prevailed in the two tests and thus the differences in tracer responses

\section{Multiple Single-Well Injection-Withdrawal Tracer Tests}

The first tracer method involved conducting two single-well injection withdrawal (SWIW) tracer tests in well $22 \mathrm{~S}$ that were identical in every respect except for the time that was allowed to elapse between the end of tracer injection and the initiation of pumping; i.e., the so-called "rest" or "shut-in" period. Two conservative (nonreactive) tracers were used in both tests, a halide (bromide or iodide) and a fluorinated benzoate. These tracers were selected for their wellknown nonreactive transport behavior (see Bowman (1984) for discussion of nonreactive nature of fluorinated benzoates) and also for their known difference of about a factor of 3 in aqueous diffusion coefficient, which allowed any effects of diffusion between flowing and stagnant (or near-stagnant) water to be readily interrogated from differences in the tracer responses. The rest periods in the two tests were $\sim 3$ days and $\sim 30$ day after the injection of "chase" water, a large volume of untraced water that followed the tracer solution to push tracers out of the borehole and into the surrounding aquifer $(\sim 76,000 \mathrm{~L}$ of chase water was injected after $5,700 \mathrm{~L}$ of tracer solution). The chase water was intended to minimize the influence of flow distortion in the aquifer caused by the presence of the borehole and also to interrogate a larger volume of the aquifer, thus providing a more representative estimate of the flow velocity in the alluvium. Further details of the test procedure are provided in Reimus et al. (2006). 
The ambient groundwater velocity was estimated by attributing the differences in the peak, mean, and late arrival times of the tracers in the two tests to the "drift" of tracers under ambient flow conditions during the extra 27 days that the tracers resided in the flow system during the test with the 30-day rest period (after accounting for radial flow during pumping and any effects of diffusion into stagnant pore water). The test results and estimated groundwater velocities have previously been reported (Reimus et al., 2006), and the details of the methods used to estimate groundwater velocity from the tracer breakthrough curves are provided in Reimus et al. (2003a). In this paper, the resulting flow velocity estimates from the SWIW tests are simply listed in Table 1 (Results section). We note that the conservative tracers with different diffusion coefficients had identical normalized responses (concentrations divided by injection masses) during the pumping/recovery period in each single-well test, which indicated that diffusion into stagnant or secondary porosity did not have a significant influence on the transport behavior of the tracers and thus did not have to be accounted for when estimating the groundwater velocity

from the tracer responses. The spreading of the tracer mass evident in the responses instead was attributed to dispersion processes resulting from the tracer being initially distributed into flow pathways of varying velocities.

\section{Tracer Responses after Flow Interruption in Forced-Gradient Test}

A forced-gradient, cross-hole tracer test involving tracer injections into wells 22PA and 22PC while pumping well $22 \mathrm{~S}$ at a constant rate was initiated in January 2005. Among the tracers injected were two fluorinated benzoates (one in each injection well), which had no measurable background concentrations in the aquifer prior to testing. The tracers were dissolved in $\sim 1000 \mathrm{~L}$ of water and the tracer solution was followed by $\sim 260 \mathrm{~L}$ of untraced chase water, which was approximately twice the dead volume of the borehole plus filter pack in the injection 
interval. In March 2005, the test was terminated after high-quality breakthrough curves had been obtained for the tracers injected into both injection wells. The results and interpretations of this test are discussed in detail in Reimus et al. (2006). 159 days after the test was terminated, in August 2005, the pumping of $22 \mathrm{~S}$ was resumed for approximately two weeks to determine residual tracer concentrations in the vicinity of $22 \mathrm{~S}$ and also to prepare for a second forcedgradient cross-hole tracer test.

During the 159 days of no pumping, the residual tracer mass remaining in the aquifer "drifted" with the ambient groundwater flow in the alluvium. The head data in the vicinity of Site 22 and other lines of evidence indicated that the natural gradient was roughly oriented from 22PA to $22 \mathrm{~S}$, so the residual tracer "plumes" at the beginning and end of the 159-day "shut-in" period were expected to appear approximately as depicted as in Figure 3. These expectations were qualitatively confirmed by the observation of higher concentrations of tracers injected into 22PA upon resumption of pumping of $22 \mathrm{~S}$ (relative to the concentrations when pumping had ceased in March) and a lower concentration of the tracer injected into 22PC. Figure 4 shows the concentrations of the fluorinated benzoates from each injection well for the first 200 hours after pumping resumed relative to their concentrations prior to the stoppage of pumping. Assuming a 22PA-to-22S orientation of the gradient, the responses of the tracers that had been injected into 22PA upon resumption of pumping we used to estimate the ambient groundwater velocity as follows:

(1) Assuming a southerly translation of a wedge-shaped plume of relatively constant concentration during the 159 days of no pumping and then radial flow upon resumption of pumping, the relative tracer concentration in the pumped water as a function of time can be estimated from simple geometric calculations. The tracer concentration in each concentric 
ring of aquifer water produced by radial convergent flow will be proportional to the fraction of the ring area that overlaps the wedge-shaped plume when pumping resumes (Figure 5). In contrast, the tracer concentration that would have been produced in each concentric ring if pumping had not been interrupted is a constant proportional to the angle at the apex of the pie slice divided by $2 \pi$ or 360 degrees. Importantly, although the absolute concentrations produced in the concentric rings under either scenario depend on the assumed angle and the assumed concentration in the plume, the ratio of the concentrations in the two scenarios isindependent of the angle assumed or the plume concentration. This ratio as a function of volume produced is dependent only on the distance that the plume translates during the 159 days of ambient flow.

(2) The times at which each concentric ring of water is produced can be estimated from $\left(\mathrm{r}^{2} / 16.7^{2}\right)$ $X 200$ hrs, where $r$ is the radius of the ring in meters. This expression assumes radial flow at the same rate before and after the flow interruption (which was approximately the case), with $16.7 \mathrm{~m}$ being the distance between wells 22PA and 22S, and $200 \mathrm{hrs}$ being the time to peak concentration of tracers injected into 22PA during pumping of 22S in January 2005. Note that while this approach assumes radial flow, a flow porosity estimate is not needed because the travel times associated with each concentric ring are scaled based on the tracer travel times observed under forced-gradient conditions.

(3) The additional mass of tracer recovered after resumption of pumping relative to what would have been recovered without the flow interruption can be readily calculated from the area under an artificial breakthrough curve obtained by subtracting the tracer concentration at the end of pumping in March from the concentrations observed after pumping resumed (once this difference becomes negative, no more additional mass is recovered - see Figure 4). 
(4) For different assumed translations of the plume during the 159 days of ambient flow, different additional mass recoveries will be calculated. The assumed translation that provides the best match to the additional recovery calculated from the actual tracer breakthrough curves is taken to be the best estimate of translation, and this estimate divided by 159 days provides an estimate of the ambient flow velocity

This approach is a relatively straightforward, albeit somewhat tedious, exercise in geometry and trigonometry that we do not describe in detail here. We simply note that the calculations involve determining both the areas in orange and blue within each concentric ring of Figure 5, with the areas in blue being more challenging to calculate than the orange wedges, which are just simple fractions of the ring areas. Note that matching the additional tracer recovery after resumption of pumping provides a more robust estimate of flow velocity than matching to a concentration history because mass recovery is an integrated measure of plume translation that is less sensitive to the assumed shape of the plume than the concentration history. The method assumes that there is no background translation of the plume after pumping resumes (i.e., no superposition of the natural gradient velocity on the velocities induced by pumping). If the additional mass is recovered in a relatively short time compared to the 159-day quiescent period (if the concentrations remain greater than the concentration at the end of pumping in March 2005 for only a short time), say a week or so, this assumption should introduce very little error.

The wedge shape of the plume is clearly a simplifying assumption, but this shape would be expected if flow was approximately radial during pumping and if the tracer solution were uniformly distributed in a cylinder about the injection well after the injection of the chase water that followed the tracer. The assumption of a constant concentration within the plume is also a 
simplifying assumption, but it is consistent with the observation that concentrations had leveled off to nearly constant values when pumping ceased in March 2005.

Finally, the method also assumes translation of the plume in exactly the direction from the injection well to the production well. In reality, it is much more likely that the ambient flow direction will be at some oblique angle relative to the line connecting the two wells. For small angles, the error in the velocity estimate from the method will be relatively small, but it will increase as the angle increases. However, the angle cannot be too great before the concentration upon resumption of pumping is no longer predicted to exceed the concentration when pumping stopped. Given that only $\sim 1260 \mathrm{~L}$ of water was injected into 22PA for the cross-hole tracer test (1000 L of tracer solution followed by $\sim 260 \mathrm{~L}$ of untraced chase water), the plume would be expected to be quite narrow. $1300 \mathrm{~L}$ of water injected over the $30.1 \mathrm{~m}$ interval thickness into a homogeneous, isotropic medium with a flow porosity of 0.1 would result in a cylinder-shaped initial distribution of tracer around the injection well with a radius of about $0.37 \mathrm{~m}$. Even if the tracer were injected into only $1 / 10^{\text {th }}$ of the interval thickness (due to layered heterogeneity), the radius of the initial cylinder would only be about $1.2 \mathrm{~m}$ (note that logging of specific conductance up and down the injection interval for $\sim 20 \mathrm{hrs}$ after tracer injection indicated that the tracer concentrations declined quite uniformly over the length of the interval, which argues against strongly-layered heterogeneity - see SNL 2007). The resulting wedge-shaped plume would have an angle at its apex of about 2.5 degrees in the former case and about 8 degrees in the latter case. In either case, a relatively narrow plume is predicted, suggesting that the natural flow direction must not deviate substantially from the line directly connecting the two wells if the tracer concentration upon resumption of pumping is initially higher than when pumping stopped. Of course, in a more realistic heterogeneous medium, the tracer flow pathway(s) 
between the two wells during pumping may have been quite circuitous, resulting in the tracer converging on the production well at a significant angle from the line connecting the two wells.

\section{Multiple Tracer Decay Rates in Injection Well With and Without Downgradient Pumping}

During and immediately after the injection of the tracers into well 22PA in both the forcedgradient cross-hole test mentioned in the previous section (Reimus et al., 2006) and a natural gradient tracer test discussed in the next section, the specific conductance in the injection interval was logged to monitor the decline in tracer concentrations in the borehole after injection. These declines could be interpreted as a borehole dilution test (Drost et al., 1968; Freeze and Cherry 1979) to obtain an estimate of the ambient flow velocity in the vicinity of 22PA. However, because the tracer solution was "chased" out of the borehole in each test by the injection of a small amount of tracer-free water (about 2 times the borehole plus filter pack volume), the assumption of a uniform distribution of tracer confined to the borehole after injection (necessary for a borehole dilution test interpretation) was not satisfied. Furthermore, there was no way to keep the injection interval mixed in the 2 -inch piezometer, so this additional assumption of a borehole dilution test was also not satisfied.

The key to estimating local ambient groundwater velocity therefore was to compare the decline of tracer concentrations under natural gradient conditions with the decline in concentrations while pumping the downgradient well $22 \mathrm{~S}$ during the forced-gradient test. In both tests, exactly the same volumes of tracer solution and chase water were injected into well 22PA (and at approximately the same rates), so the initial distribution of tracers around 22PA should have been nearly identical (even solution density contrasts were kept almost the same). The greater decline in tracer concentrations during the forced-gradient test could thus be attributed to the influence of pumping from well 22S. Furthermore, the local flow velocity at 
22PA induced by the pumping of well $22 \mathrm{~S}$ could be estimated from the tracer travel times in the forced-gradient test, with the assumption that radial flow rates varied with $1 / r$ (i.e., radial flow) and the natural flow velocity was negligible. In this case, the following equation is valid:

$$
\tau=\int_{r_{w}}^{R} \frac{d r}{V(r)}=\int_{r_{w}}^{R} \frac{d r}{(K / r)}=\int_{r_{w}}^{R} \frac{r d r}{K}=\frac{1}{2 K}\left(R^{2}-r_{w}{ }^{2}\right)
$$

where $\tau=$ mean tracer travel time in forced-gradient tracer test, $\mathrm{yr}$

$r=$ radial coordinate, $\mathrm{m}$

$V(r)=K / r=$ radial velocity as a function of $r, \mathrm{~m} / \mathrm{yr}$

$R=$ distance between injection and pumping well, $\mathrm{m}$

$r_{w}=$ radius of pumping well, $\mathrm{m}$

$K=$ constant with units of $\mathrm{m}^{2} / \mathrm{yr}$.

For ideal radial flow in a homogeneous, isotropic medium, $K=Q / 2 \pi b \phi$, where $Q=$ pumping rate $\left(\mathrm{m}^{3} / \mathrm{yr}\right), b=$ interval thickness $(\mathrm{m})$, and $\phi=$ flow porosity. However, equation ( 1$)$ is also true in general if the flow velocity varies as $1 / r$ (i.e., pseudo-radial flow), and the constant $K$ can be solved if the travel time $\tau$ is known, as it is in the case of the forced-gradient cross-hole test. In this case, $K=\left(R^{2}-r_{w}{ }^{2}\right) / 2 \tau$. Once $K$ is estimated, the local flow velocity at the injection well location is given by $K / R$. If flow is not approximately radial, equation (1) will take a slightly different form, but the highest possible local flow rate would be obtained assuming linear flow (i.e., a constant flow rate between the injection and production well), in which case the forcedgradient velocity would be given by $\left(R-r_{w}\right) / \tau$. Pseudo-radial flow seems a more appropriate assumption than linear flow given that flow pathways are expected to converge near the pumping well and velocities should therefore increase as distance from the pumping well decreases. However, regardless of the flow conditions assumed, the ratio of the rates at which tracer 
concentrations decline in the injection well during the natural-gradient test and in the forcedgradient test can be used as a proportionality factor to estimate the local flow velocity under natural gradient conditions. That is,

$$
V_{n a t}=\frac{(\text { tracer decay time in natural grad test })}{(\text { tracer decay time in forced grad test) }} V_{\text {forced }}
$$

where $V_{\text {nat }}=$ natural gradient velocity, $\mathrm{m} / \mathrm{yr}$

$V_{\text {forced }}=K / R=$ forced gradient velocity at injection well, $\mathrm{m} / \mathrm{yr}$.

Figure 6 shows the normalized tracer concentrations (concentrations divided by injection concentrations) in injection well 22PA during the start of the forced-gradient and naturalgradient tracer tests plotted as the natural log of the concentrations vs. time (the manner in which concentrations would be plotted for a classical borehole dilution test analysis). Both curves were obtained using a YSI 600XLM sonde (Yellow Springs, Inc.), with the specific conductance measurements measured at the midpoint depth of the screened interval converted to concentrations using background-subtracted calibration curves. In the case of the forcedgradient test, both the actual decay function and the decay function with a time scale multiplied by a factor of 26 are shown. The latter curve constitutes an adjustment so that the tail of the forced-gradient curve intersects the tail of the natural-gradient curve near the end of the time period when the natural-gradient decay curve was well-behaved (seismic activity in the area that occurred about 160 hours after the natural-gradient injection appears to have caused unexplained fluctuations in the tracer curve starting at that time). Note that the up-and-down spikes in the forced-gradient curve correspond to times when the YSI sonde was moved up and down the screened interval to obtain depth profiles of specific conductance. Figure 7 shows examples of such profiles from the natural gradient test; these serve to illustrate that vertical concentration gradients were not extreme, except perhaps late in the test. For the majority of the time, the 
probe was kept at the same depth in both tests. Neither curve of Figure 6 exhibits the classic linear trend that is expected for a plot of log concentration vs. time in a borehole dilution test (Drost et al., 1968; Freeze and Cherry 1979), which is likely because the conditions required for a borehole dilution test were not met in either test. Although the shapes of the curves are slightly different, the time-scale adjustment necessary for the two curves to have the same late-time concentration was taken to be the proportionality factor of equation (2).

Two inherent advantages of this method over the classical borehole dilution method are that it does not rely on estimates of borehole flow distortion factors (Freeze and Cherry, 1979; Palmer, 1993), and it also does not require special equipment to keep the borehole mixed during the test. It only relies on identical injection volumes of tracer solution and chase water in the two tests and on an estimate of the local flow velocity derived from a cross-hole tracer response in a forced-gradient test in which the measurement well is used as an injection well. In fact, the pushing of the tracer out of the borehole and into the aquifer might be considered advantageous from the standpoint of avoiding flow nonidealities through the borehole due to wellbore completion artifacts. Another advantage from the standpoint of estimating a natural-gradient pore velocity is that the method does not rely on an estimate of the flow porosity in the aquifer to convert from the specific discharge estimate classically obtained from a borehole dilution test to a pore velocity. The velocity derived from the mean tracer travel time in a cross-hole test is inherently a pore velocity, so the method gives a direct estimate of pore velocity without relying on a knowledge of flow porosity. Of course, it seems intuitively evident that the best estimate is likely to be obtained if the forced-gradient and natural-gradient velocities are aligned in the same direction.

\section{Natural Gradient Tracer Test}


A natural gradient tracer test (tracer test without any active pumping) was initiated on October 24, 2006 by injecting a solution containing sodium bromide and pentafluorobenzoate (PFBA), two nonreactive tracers with different molecular sizes and hence different diffusion coefficients, into well 22PA. The volumes of tracer solution and untraced chase water injected were identical to those in the earlier forced-gradient cross-hole tracer test $(\sim 1000 \mathrm{~L}$ and $\sim 260 \mathrm{~L}$, respectively). After injection of the tracer solution, small volume samples ( $230 \mathrm{~L}$ each) were periodically withdrawn from well $22 \mathrm{~S}$ and analyzed for the presence of tracers. The $\sim 230-\mathrm{L}$ volume of each sample corresponded to approximately one interval volume in 22S. Larger samples were avoided to minimize the influence of sampling on the natural flow field, and subsamples were collected after $\sim 40 \mathrm{~L}$ and $\sim 120 \mathrm{~L}$ during each sampling event to determine if there was any time dependence of concentrations during the sampling, which there was not.

Figure 8 shows the PFBA breakthrough curve in $22 \mathrm{~S}$ after nearly two years of sampling under natural flow conditions. The best-fitting model curve from the methods discussed below is also shown in this figure. The simplest approach to estimating an ambient flow velocity from this breakthrough curve is to simply divide the distance between the injection and sampling wells $(16.7 \mathrm{~m})$ by the peak or mean arrival time of the tracer. This approach implicitly assumes that the flow direction is very close to the direction of well $22 \mathrm{~S}$ from 22PA. The peak arrival time from Figure 8 is $\sim 330$ days, and the mean arrival time is $\sim 470$ days, as determined from fits of the 1-D advection-dispersion equation to the breakthrough curve using the RELAP model (Reimus et al., 2003b). The resulting flow velocity estimates are 18.5 and $13.0 \mathrm{~m} / \mathrm{yr}$, respectively. We consider the estimate based on the mean tracer arrival time to be a better estimate of the overall average flow velocity in the tracer test interval, although the highervelocity estimate based on peak arrival time could be considered a good estimate for the more 
transmissive flow pathways in the interval. No adjustments to advective travel times were considered necessary to account for delays due to tracer diffusion into stagnant water because the differences between the breakthrough curves of the PFBA and bromide (which have diffusion coefficients that differ by about a factor of 3) were insignificant. As in the case of the shorterduration single-well injection-withdrawal tests, it was concluded that diffusion into stagnant/secondary porosity was insignificant and spreading of the tracer mass was attributed to dispersion resulting from the tracers being injected into and moving through multiple flow pathways of different velocities.

\section{RESULTS}

\section{Multiple Single-Well Injection-Withdrawal Tracer Tests}

The flow velocity estimates from the analyses of the two single-well injection-withdrawal tests in well 22S are listed in Table 1. The estimates depend on whether the peak, mean, or late arrival times are used in the calculations (Reimus et al., 2003a) and also on the assumed flow porosity in the aquifer. This method is the only method discussed in this paper for which the flow velocity estimate depends on the flow porosity. The reason is that the flow velocities in the aquifer induced by pumping during the withdrawal phase of the single-well tests are dependent on the flow porosity, and these induced flow velocities in turn affect the estimates of the ambient flow velocity. We note that while Reimus et al. (2006) reported estimates only for the peak and late arrival times of tracer, Table 1 also includes estimates for the mean arrival times. These estimates were previously calculated, but they were not included in Reimus et al. (2006) because only the upper and lower bound estimates were reported in that paper. However, of the three types of estimates (peak, mean and late), we consider those based on mean arrival times to best 
represent the average flow velocity in the aquifer because they reflect the movement of the center of mass of the tracer plume. In contrast, the estimates based on peak arrival times reflect the typically slower movement associated with the highest concentration portion of the plume (when it is withdrawn) and the estimates based on late arrival times reflect the higher flow velocities that result in tailing in the tracer breakthrough curves.

The flow porosities listed in the top row of Table 1 represent, respectively, the flow porosities estimated from two forced-gradient tracer tests conducted between 22PA and 22S (Reimus et al., 2006), and an approximate average of these two flow porosities (0.1). Note that the multiple single-well tracer test method provides no information on ambient flow direction.

\section{Tracer Responses after Flow Interruption in Forced-Gradient Test}

The best match to the additional recovery of tracer mass that was injected into well 22PA in well 22S after the 159-day flow interruption in 2005 was obtained by assuming the tracer plume moved approximately $3.25 \mathrm{~m}$ during the flow interruption. Figure 9 shows the simulated and actual tracer responses expressed as a fraction of the pre-flow-interruption concentration. Although the concentration history was not matched exactly, it is apparent that it is reasonably well approximated by the simple model that assumes translation of a wedge-shaped plume in a homogeneous, isotropic medium. The 3.25-m translation of the plume in 159 days corresponds to a flow velocity of $7.5 \mathrm{~m} / \mathrm{yr}$.

One of the key assumptions and consequently key uncertainties with this estimation method is the assumption of the wedge shape of the plume. This assumption would be expected to be most suspect at greater distances from the production well, closer to the injection well, and in the most extreme case the plume might more closely approximate a fingerlike shape with nearly constant cross-section. Given that all the additional mass recovered must enter the volume 
produced during the first $\sim 160$ hours after pumping resumes by crossing the upgradient boundary of this control volume, and the fact that the peak concentration from 22PA in the forced gradient tracer test occurred $\sim 200$ hours after injection, it can be surmised that the additional tracer mass must have been pretty close to the injection well when pumping ceased in March. If the shape of the plume were approximately rectangular (or more generally had a nearly constant crosssectional area) rather than wedge shaped, then the distance that the plume would have translated during the flow interruption would be estimated to be 0.35 times the distance between the injection and production wells, or $0.35(16.7)=5.85 \mathrm{~m}$, where 0.35 is the additional tracer mass divided by the mass that would have been recovered without the flow interruption (see Figure 4). In this case, the ambient flow velocity estimate would be $12.5 \mathrm{~m} / \mathrm{yr}$. We can take this as an upper bound for the flow velocity estimated by this method. However, it should be recognized that if the plume concentration decreased as distances from the production well increased (i.e., closer to the injection well) instead of remaining constant (as assumed), which might be expected based on the very slow decline in tracer concentrations at the time pumping ceased, then the ambient flow velocity required to account for the additional tracer mass would be slightly larger than the estimates of 7.5 and $12.5 \mathrm{~m} / \mathrm{yr}$ given above. For this reason, we give more weight to the larger estimate of $12.5 \mathrm{~m} / \mathrm{yr}$ obtained by assuming a constant cross-sectional area of the upgradient tracer plume.

We intuitively expect that flow velocity estimates obtained from this method will be lower than by other methods because, as discussed above, the additional tracer mass recovered is expected to be primarily mass lingered near the injection well before pumping ceased. The fact that this mass lingered near the injection well and was not recovered when the majority of the injected tracer mass was recovered by the time pumping stopped in March 2005 suggests that 
this mass managed to get into low velocity (low permeability) regions of the aquifer, even under forced-gradient conditions. Any estimate of flow velocity based on interrogating tracer mass that initially resides in low velocity regions of an aquifer is bound to be biased low.

\section{Multiple Tracer Decay Rates in Injection Well With and Without Downgradient Pumping}

The mean tracer arrival time in the second forced-gradient cross-hole tracer test between wells 22PA and 22S was estimated to be 215 hours (Reimus et al., 2006), and the distance between the wells is 16.7 meters. Substitution of these values into equation (1) (assuming $r_{w}$ is negligible) and solving for $K$ yields $K=0.649 \mathrm{~m}^{2} / \mathrm{hr}$. The estimate of local flow velocity at well 22PA during this test is therefore $0.649 / 16.7=0.039 \mathrm{~m} / \mathrm{hr}$, and the best estimate of naturalgradient velocity would be a factor of 26 less than this, or $13.1 \mathrm{~m} / \mathrm{yr}$. If the flow during the forced-gradient test is assumed to be linear rather than pseudo-radial, then the local flow velocity during the cross-hole test would be estimated to be $16.7 / 215=0.078 \mathrm{~m} / \mathrm{hr}$, and the best estimate of natural-gradient velocity would be a factor of 26 less than this, or $26.2 \mathrm{~m} / \mathrm{yr}$. Thus, the estimates from the multiple tracer decay method range from 13.1 to $26.2 \mathrm{~m} / \mathrm{yr}$, depending on the type of flow assumed during the forced-gradient tracer test. We believe that the lower estimate, which assumes pseudo-radial flow, is a better estimate because it seems unlikely that the flow velocity as a function of radial distance from the production well in the forced gradient test was constant.

\section{DISCUSSION}

Table 2 summarizes the groundwater velocity estimates from each of the tracer methods described in the preceding sections. A flow porosity of 0.1 is assumed for the multiple singlewell tracer test method because 0.1 is approximately the average of the flow porosity estimates 
from the two forced-gradient cross-hole tracer tests conducted between wells 22PA and 22S.

These are considered to be the best available estimates of local flow porosity in this flow direction. The agreement between the different methods is reasonably good, and it is excellent when one considers only the bold values in Table 2, which are thought to be the best estimates of average flow velocity within the test interval (see preceding sections).

The only independent, non-tracer-based estimates of flow velocity that can be provided for comparison with the tracer methods are those obtained by multiplying the hydraulic conductivity estimated from pump tests in the tracer test interval at Site 22 by natural gradient estimates from water level measurements at Site 22 and in wells located several $\mathrm{km}$ up and down gradient from Site 22. The potentiometric surface is too flat to obtain estimates of the hydraulic gradient from water level differences in wells located only at Site 22. The flow velocity estimates calculated from hydraulic gradients determined from different combinations of wells and assuming a hydraulic conductivity of $12 \mathrm{~m} /$ day based on pump test data (SNL, 2007) are provided in Table 3 , and they range from about 30 to $120 \mathrm{~m} /$ year. These estimates tend to be higher than the tracer-based estimates of Table 2, but they are also probably more uncertain because the gradient estimates were obtained over much larger scales (several $\mathrm{km}$ ) than the local scale of the tracer tests $(\leq 20 \mathrm{~m})$. It is also possible that the local gradient in the immediate vicinity of Site 22 is much flatter than the larger-scale gradient extending over several km. Also, heads in the alluvium are known to increase with depth (upward vertical gradient), so different water levels at different locations might reflect differences in well penetrations and/or differences in layered heterogeneity at the different locations rather than true potentiometric surface differences that can be applied to the tracer test interval. For all these reasons, we do not consider the flow 
velocity estimates based on hydraulic gradient and hydraulic conductivity estimates to be as good as the estimates from the tracer-based methods, at least at the local scale of the tracer tests.

Appendix A describes two alternative approaches that were used to evaluate uncertainties and nonuniqueness in the ambient flow velocity estimates from the tracer breakthrough curve in the natural-gradient tracer test as a result of not knowing the exact flow direction relative to the direction from well 22PA to well 22S. Both approaches involve matching the observed breakthrough curve by assuming three-dimensional advection and Gaussian dispersion of an initial distribution of tracer mass near well 22PA through a homogeneous, isotropic medium to the vicinity of $22 \mathrm{~S}$. The models tended to significantly overpredict the observed tracer concentrations, so they compensated by either using a very large transverse dispersivity (if tracer migration was forced to be almost directly from well 22PA to $22 \mathrm{~S}$ ), or alternatively, if the transverse dispersivity was constrained to have more reasonable values, it was necessary to specify a flow direction that was at a significant angle from the most direct path between wells 22PA and 22S. In the latter case, the estimated flow velocities in the principle direction of flow were all significantly larger than the estimates provided in Table 2 .

While the results of Appendix A cannot be ruled out as being invalid, it seems much more plausible that the alluvium at Site 22 did not conform to the homogeneous, isotropic medium assumptions used in the calculations. Instead, it is likely that the flow direction was very close to the most direct path between wells 22PA and 22S, and local heterogeneities simply caused the highest tracer concentrations to miss $22 \mathrm{~S}$. Thus, we take the flow velocity estimates provided in this section as being the most representative of the true velocity at Site 22, and we use Appendix A primarily to illustrate the uncertainty and nonuniqueness in velocity estimates when a homogeneous, isotropic medium is assumed. The implication here is that assuming a 
homogeneous, istotropic medium actually seems to be counterproductive for estimating a flow velocity in the case of having only one downgradient observation well. In hindsight, given the very short distance into the aquifer that the tracer solution was injected (calculated to be only $\sim 0.37 \mathrm{~m}$ beyond the 22PA filter pack assuming uniform radial flow and a flow porosity of 0.1 ), it might be considered quite remarkable that tracer was observed at all in well 22S. The fact that tracer was observed seems to strengthen the argument that the flow direction was probably very well aligned with the direction from 22PA to $22 \mathrm{~S}$.

It is of practical interest to compare and contrast the relative merits and disadvantages of the different flow velocity estimation methods represented in Table 2 . We divide this discussion into two parts, covering the single-well method first and then the two cross-hole methods (the natural-gradient test method might be considered a third cross-hole method, but it is taken here to provide a ground-truth estimate).

\section{Single-Well Method}

The multiple single-well tracer test method is really the only true single-well test method that was employed in this paper. This method has a significant advantage over the cross-hole test methods in that only one well is needed to obtain a groundwater flow velocity estimate. Although well drilling and completion costs vary greatly in different hydrogeologic settings, wells are generally among the highest expenditures in any hydrogeological characterization program, so reducing the number needed to obtain a velocity estimate can result in significant savings, or alternatively, it can allow measurements at more spatial locations for the same budget. However, as previously discussed, the single-well method provides no information on flow direction, and groundwater velocity estimates from single-well tests are dependent on the flow porosity assumed, which can only be estimated effectively from cross-hole tests. In 
addition, single-well tests interrogate a smaller volume than cross-hole tests and thus provide information on groundwater flow at a smaller spatial scale. While it is possible that some flow direction information could be obtained using directional flow meters in a single well, these measurements could be compromised by numerous potential nonidealities that may cause the flow within the borehole to be distorted relative to the natural gradient flow.

\section{Cross-Hole Methods}

In addition to the natural-gradient tracer test, which is considered here to provide a groundtruth estimate of the natural flow velocity, the cross-hole flow interruption method and the multiple tracer decay method are the two cross-hole methods used in this study to estimate groundwater flow velocity. The multiple tracer decay test is considered a cross-hole method here because it requires a forced-gradient cross-hole tracer test to be implemented.

The primary advantage that the cross-hole flow interruption method offers over the other methods is that it can provide information on flow direction in the aquifer in addition to flow velocity. However, this advantage may not be significant in settings where the flow direction is considered to be well understood (i.e., where gradient direction is well-established or obvious). The other advantage that the cross-hole flow interruption method offers over the other methods is that the volume of aquifer interrogated is much larger than in a single-well test or in a multiple tracer dcay test. Even for the large volumes of tracer and chase water used in the single-well tracer tests conducted at Site $22(\sim 80,000 \mathrm{~L})$, the average radial tracer penetration distance into the aquifer was estimated to be only $\sim 3 \mathrm{~m}$ (largely due to the long interval), assuming a homogeneous aquifer and a flow porosity of 0.1 . In contrast, the cross-hole test interrogation scale can be taken to be the distance between the injection and observation wells, or $\sim 17 \mathrm{~m}$ at Site 22. All other things being equal, flow velocity estimates based on larger interrogation 
volumes or larger scales of measurement are generally considered more applicable for risk assessment predictions than smaller scale measurements. Another advantage of the cross-hole flow interruption method is that a flow porosity estimate can be obtained from the tracer breakthrough curve(s) prior to the flow interruption.

The multiple tracer decay method is best implemented as a low-cost add-on to a forcedgradient cross-hole tracer test, but it requires that the tracer injection and chase water volumes are kept exactly the same in both tracer injections. A forced-gradient test by itself does not provide an estimate of natural flow velocity, but by observing tracer concentration declines under both forced and natural gradient conditions, a reasonable estimate of natural flow velocity can be obtained. The advantages of the multiple tracer decay method over a classical borehole dilution test method (which is a true single-well method), are discussed in the Methods section (i.e., it does not require an estimate of the borehole flow distortion factor, and it allows a more reliable estimate of the pore velocity because the cross-hole forced-gradient tracer response yields a flow porosity estimate to convert specific discharge to pore velocity).

Both cross-hole tracer test methods have disadvantages. As previously mentioned, the flow interruption method is expected to result in flow velocity estimates that are biased low because it is based on the movement of tracer mass corresponding to the tail of a forced-gradient tracer test, which is mass that has likely lingered in low-velocity regions in the aquifer. This bias problem might be lessened by interrupting flow earlier in a forced-gradient test, but this could compromise other objectives of the forced-gradient test, and the interpretation of the tracer response after the flow interruption will be more uncertain if the interruption occurs when tracer concentrations are changing significantly during the forced-gradient test. Also, the flow interruption method relies on an ambient flow direction that is quite closely aligned with the flow 
direction induced by pumping prior to the flow interruption. Although it was not addressed in this paper, the uncertainties associated with flow velocity estimates when the ambient flow direction is not well aligned with the forced-gradient flow direction are expected to be quite large and will likely limit the method to very qualitative estimates of flow velocity and direction. However, scoping calculations suggest that if the flow direction is within 20 to 25 degrees or so of the alignment of the wells, the flow velocity estimates should be reasonably accurate.

Whenever possible, the use of multiple injection wells, as at Site 22, can help constrain estimates of flow direction and thus flow velocity.

The multiple tracer decay method has the disadvantage that, similar to the single-well injection-withdrawal method, it interrogates only a small volume of the aquifer in the immediate vicinity of the injection well. However, it relies on observing a tracer concentration decline in the injection borehole during a forced-gradient test to obtain a natural-gradient velocity estimate, so in that sense it uses a result obtained at a larger scale. If the natural flow velocity is a significant fraction of the flow velocity induced during the forced-gradient test, then it may be necessary to make a correction to account for the superposition of the natural gradient velocity on the forced-gradient velocity. This will require a modification to equation (1) and the simultaneous solution of the following two equations to obtain a velocity estimate:

$$
\tau=\int_{r_{w}}^{R}\left(\frac{1}{V(r)+V_{n a t}}\right) d r=\int_{r_{w}}^{R}\left(\frac{1}{(K / r)+V_{n a t}}\right) d r=\frac{\left(R-r_{w}\right)}{V_{n a t}}+\frac{K}{V_{n a t}^{2}} \operatorname{Ln}\left(\frac{V_{n a t} r_{w}+K}{V_{n a t} R+K}\right)
$$

and

$$
\frac{K / R+V_{n a t}}{V_{n a t}}=\frac{(\text { tracer decay time in natural grad test })}{(\text { tracer decay time in forced grad test })}
$$

In this case, equations (3) and (4) would be solved simultaneously to obtain estimates of $K$ and $V_{\text {nat }}$, with the latter being the natural gradient velocity. If the forced-gradient flow is assumed to 
be linear (not pseudo-radial), equation (3) simply becomes $\tau=\left(R-r_{w}\right) /\left(K+V_{\text {nat }}\right)$, and $K / R$ will be replaced by $K$ in equation (4). Equation (1) was used in this paper to estimate the natural gradient velocity at Site 22 because it was recognized that the natural flow velocity was very small compared to the forced-gradient velocity, in which case equation (3) reduces to equation (1). Scoping calculations suggest that if the ratio of observed tracer decay times (in equations (2) and (4)) exceeds about 15 or 20, then equations (1) and (2) provide a very good estimate of the natural flow velocity.

We note that it should be particularly advantageous to conduct both a cross-hole flow interruption test and a multiple tracer decay test during the same testing campaign because the former is biased toward interrogating slower flow pathways and thus should provide a lowerbound estimate of natural flow velocities, while the latter should provide more of an upper bound estimate because it tends to be influenced more by faster flow pathways that intersect the injection borehole. Additionally, the multiple tracer decay measurement reflects velocities near the injection well, while the flow-interruption measurement reflects velocities near the production well. Thus, the two combined estimates inherently address spatial variability in natural flow velocities at a site. A tracer decay test under natural gradient conditions could easily be conducted during the flow interruption phase of the cross-hole test.

Although natural gradient tests are inherently the best method for estimating ambient flow velocity and direction, a natural gradient test carries with it considerable risk that the observation well(s) will not intersect the tracer plume at all, resulting in no useful information other than ruling out flow directions. This risk decreases as the number of observation wells increases, but costs also increase proportionately with the number of wells, and costs can quickly become prohibitive in deep environments such as Site 22. Natural gradient tests also carry the risk of 
potentially long and unpredictable test durations, although at least the costs of conducting natural gradient tests are decreased by not having to pump or maintain power at the site.

\section{CONCLUSIONS}

The array of tracer tests at Nye County Site 22 offer a unique opportunity to compare and contrast three different tracer methods for estimating groundwater flow velocity, with a natural gradient tracer test serving as a fourth method that provided a "ground truth" estimate. The data sets analyzed in this paper are especially rare for a hydrogeologic setting with a deep water table where well drilling and completion costs are significant. The methods are in reasonably good agreement (Table 2) considering the different scales of interrogation and different limitations of the methods, as discussed in the previous sections. We consider the results of this study to be particularly applicable to sites where the water table is deep and is thus expensive to access, and also where natural gradients are flat so that natural flow velocities are quite uncertain. This study is less applicable to situations were numerous wells can be installed (e.g., shallow environments) because natural flow velocities can then be determined relatively inexpensively and without significant risk by the ground-truth natural gradient tracer test method.

The multiple single-well tracer test method is attractive in that the volume of interrogation can be controlled to some extent and only one well is required. Although it does not provide flow direction information and relies on an assumed flow porosity, the flow direction and flow porosity are often reasonably well constrained by other data. In almost all technical respects, multiple single-well tests should be superior to simple borehole dilution tests (Drost et al., 1968; Freeze and Cherry 1979), although the latter can be conducted more quickly and inexpensively. 
When multiple wells are available, the cross-hole flow interruption method in conjunction with the multiple tracer decay method (one measurement conducted under forced-gradient conditions, the other under natural-gradient conditions during the flow interruption) is recommended as a relatively inexpensive add-on to any forced-gradient cross-hole tracer test that is aligned reasonably well with the natural flow direction. The natural flow velocity estimates

obtained by these two methods are complimentary in that they should constitute lower and upper bound estimates, respectively, and they also are obtained in different spatial locations in the aquifer (near the production and injection wells, respectively), which allows spatial variability in the flow velocity estimates to be evaluated. Flow direction information also can be obtained from the cross-hole flow interruption method if different tracers are injected into multiple injection wells that are oriented in different directions from the pumping well. Each tracer will have a different response after the flow interruption, and these responses will be indicative of the flow direction during the interruption.

\section{ACKNOWLEDGMENTS}

This work was supported by the U.S. Department of Energy Office of Civilian Radioactive Waste Management. The tracer tests were a collective effort of too many people to acknowledge by name from the following organizations: Nye County Department of Natural Resources (Early Warning Drilling Program) and their subcontractors, U.S. Department of Energy Yucca Mountain Project Office, Los Alamos National Laboratory, Sandia National Laboratories, U.S. Geological Survey, and the University of Nevada-Las Vegas. Los Alamos National Laboratory is managed and operated by Los Alamos National Security, LLC for the U.S. Department of Energy's National Nuclear Security Administration under contract DE-AC52-06NA25396. 
Sandia National Laboratories is a multi-program laboratory managed and operated by Sandia Corporation, a wholly owned subsidiary of Lockheed Martin Corporation, for the U.S. Department of Energy's National Nuclear Security Administration under contract DE-AC0494AL85000.

\section{REFERENCES}

Arnold, Bill W., Teklu Hadgu., and Cedric J.M. Sallaberry, 2008, Sensitivity analyses of radionuclide transport in the saturated zone at Yucca Mountain, Nevada, $12^{\text {th }}$ International High-Level Radioactive Waste Management Conference, September 7-11, 2008, Las Vegas, NV, American Nuclear Society, La Grange Park, Illinois.

Bowman, R.S. 1984. Evaluation of Some New Tracers for Soil Water Studies, Soil Sci. Soc. Am. J., 48, 987-993.

Doherty, John E., 2007, Manual for PEST: Model Independent Parameter Estimation, Watermark Numerical Computing, Brisbane, Australia.

Drost, Walter, Dietmar Klotz, Arnd Koch, Heribert Moser, Ferdinand Neumaier, and Werner Raurert, 1968, "Point Dilution Methods of Investigating Ground Water Flow by Means of Radisotopes," Water Resources Research, 4, (1), 125-146.

Freeze, R. Allan and John A. Cherry, 1979, Groundwater, Prentice-Hall, Englewood Cliffs, New Jersey.

Leij, Feike J. and Scott A. Bradford, 1994, 3DADE: A Computer Program for Evaluating ThreeDimensional Equilibrium Solute Transport in Porous Media, Research Report No. 134, U.S. Salinity Laboratory, Agricultural Research Service, U.S. Department of Agriculture, Riverside, California. 
Ogilvi, N. A., 1958, “An electrolyrical method of determining the filtration velocity of underground Waters" (in Russian), Bull. Sci.-Tech. Inf., No. 4 (16), Gosgeoltekhizdat, Moscow, 1958.

Palmer, C. D. 1993. Borehole dilution tests in the vicinity of an extraction well, J. Hydrology, $146,245-266$.

Reimus, P., M.J. Umari, R. Roback, J. Earle, J. Darnell, and I. Farnham. 2003a. Estimates of Ambient Ground-Water Velocity in the Alluvium South of Yucca Mountain, Nye County, Nevada, from Single-Well Tracer Tests, Proceedings of the $10^{\text {th }}$ International High-Level Radioactive Waste Management Conference, American Nuclear Society, pp. 102-113.

Reimus, P. W., G. Pohll, T. Mihevc, J. Chapman, L. Papelis, B. Lyles, S. Kosinski, R. Niswonger, and P. Sanders. 2003b. "Testing and Parameterizing a Conceptual Model for Radionuclide Transport in a Fractured Granite using Multiple Tracers in a Forced-Gradient Test”, Water Resources Research, 39(12), 1350, doi:10.1029/2002WR001597.

Reimus, P., Jones, R., Wasson, E., Kelly, J., Sanchez, A., Slack, W., and Daniels, J. 2006. "Results of Tracer Testing in the Saturated Alluvium South of Yucca Mountain," Proceedings of the 11th International High Level Radioactive Waste Management Conference, IHLRWM, Las Vegas, NV, April 30-May 4, 2006, pp. 315-322, American Nuclear Society, LaGrange Park, IL.

Sandia National Laboratories (SNL). 2005. Saturated Zone Flow and Transport Model Abstraction, MDL-NBS-HS-000021, Rev 03, Sandia National Laboratories, Albuquerque, NM. Available at http://www.lsnnet.gov, LSN \# DN2002451859. 
Sandia National Laboratories (SNL). 2007. Saturated Zone In-Situ Testing, ANL-NBS-HS000039, Rev 02, Sandia National Laboratories, Albuquerque, NM. Available at http://www.lsnnet.gov, LSN \# DN2002481550. 


\section{APPENDIX A: Uncertainty in Natural Flow Velocity Estimates from the Natural Gradient Tracer Test when Assuming a Homogeneous, Isotropic Medium}

The natural flow velocity estimates of 18.5 and $13.0 \mathrm{~m} / \mathrm{yr}$, obtained by dividing the $16.7 \mathrm{~m}$ distance between wells $22 \mathrm{PA}$ and $22 \mathrm{~S}$ by either the peak or mean arrival time, respectively, of the PFBA tracer in well 22S, are considered to be the best estimates of natural flow velocity at Nye County Site 22. In this appendix, we use two different methods to estimate the natural flow velocity and flow direction if it is assumed that the aquifer at Site 22 is perfectly homogeneous and isotropic, and we also evaluate the uncertainty and nonuniqueness in these estimates.

\section{Methods}

In the first method, a semi-analytical solution for advective-dispersive transport in a homogeneous medium was implemented using the 3DADE software code (Leij and Bradford, 1994). The tracer mass was assumed to be initially distributed in a rectangular block centered around the injection well with an initial concentration equal to the tracer mass divided by 1260 $\mathrm{L}$, the injection plus chase solution volume. Assuming an average porosity of 0.2 in the vicinity of the wellbore, the rectangular block was calculated to have a half-width of $0.23 \mathrm{~m}$ in the $\mathrm{x}$ and $\mathrm{y}$ directions and $30.1 \mathrm{~m}$ in the $\mathrm{z}$ (vertical) direction. Porosity is not specified as an input to 3DADE, so the porosity assumed when calculating the source volume effectively becomes the assumed flow porosity for the entire domain in the calculations. The tracer concentrations were calculated at a distance of $16.7 \mathrm{~m}$ from of the observation well and at a given distance transverse to the axis of the tracer plume.

3DADE was coupled with the PEST software code (Doherty, 2007) for parameter estimation and uncertainty analyses. PEST uses non-linear parameter estimation to obtain the model 
parameter values that result in an optimal fit between model predictions and observed data. In this case the model predictions are the simulated tracer concentrations as a function of time at the observation well and the observed data are the PFBA concentrations in well 22S. The optimization analysis with PEST involved estimation of four parameters: (1) groundwater flow velocity, (2) transverse distance of well $22 \mathrm{~S}$ from the centerline of the tracer plume, (3) longitudinal dispersivity, and (4) horizontal transverse dispersivity. The vertical transverse dispersivity was held constant at a relatively low value because the thick injection interval (about $30 \mathrm{~m}$ ) renders the test results insensitive to vertical transverse dispersivity. The objective function of the PEST optimization is the sum of equally weighted squared residuals in tracer concentration. Flow porosity was assumed constant at 0.2 in all simulations.

For the second method, a Microsoft Excel spreadsheet was set up as an alternative to the 3DADE solution. The spreadsheet made use of the NORMDIST (normal distribution) function to distribute tracer mass over a 2-D domain of specified thickness (in the $3^{\text {rd }}$ dimension) in accordance with a 2-D Gaussian distribution. The centroid of the tracer "plume" is translated with time by multiplying time by a specified velocity, and the distribution of tracer mass about the centroid in each orthogonal direction is then determined using the NORMDIST function. For any given $x$ and $y$ coordinate (with $\mathrm{x}$ being the flow direction and $\mathrm{y}$ being perpendicular to the flow direction), a relative tracer concentration is determined as follows:

(1) $\operatorname{NORMDIST}\left(x, V t, \sqrt{2 \alpha_{x} V t}\right)$ is used to obtain a concentration dilution factor (probability associated with the $x$ value) at a specified time in the direction of flow. In this case, the distribution mean is specified as $V t$, and the standard deviation is specified as $\sqrt{2 \alpha_{x} V t}$, where $\alpha_{x}$ is the dispersivity in the $x$ direction. 
(2) $\operatorname{NORMDIST}\left(y, 0, \sqrt{2 \alpha_{y} V t}\right)$ is used to obtain a concentration dilution factor (probability associated with the $y$ value) at a specified time in the direction of transverse to flow. In this case, the distribution mean is specified as zero, and the standard deviation is specified as $\sqrt{2 \alpha_{y} V t}$, where $\alpha_{y}$ is the dispersivity in the $y$ direction.

(3) The two probabilities (values returned from NORMDIST above) are multiplied to yield a dilution factor for calculating a normalized concentration (the concentration relative to the assumed injection mass) at the specified $(x, y)$ coordinate.

(4) This factor is then divided by the product of the thickness in the $z$ direction and the aquifer flow porosity to yield a normalized concentration, which must be multiplied by the injection mass to yield an absolute concentration.

The spreadsheet solution was used to fit the PFBA breakthrough curve by using the Excel SOLVER function, which minimizes a specified objective function by adjusting input parameters that are entered in specified spreadsheet cells. The objective function was the sum of squares differences between the data and the spreadsheet solution, and the adjustable input parameters were the longitudinal dispesivity, the transverse dispersivity, and the angle between the flow direction and the observation well. The flow velocity and flow porosity were fixed in any given simulation, and the interwell separation distance and interval length were kept constant for all simulations (16.7 $\mathrm{m}$ and $30.1 \mathrm{~m}$, respectively).

\section{Results}

One of the first observations made in applying either the 3DADE-PEST or the Spreadsheet methods was that the observed tracer concentrations in $22 \mathrm{~S}$ were much lower than predicted if the plume were assumed to transport directly from 22PA toward 22S in a homogeneous, isotropic medium. The only way to get the predicted and observed concentrations to match 
under these conditions was to assume an unrealistically high transverse dispersivity that served to spread out the plume enough in the direction perpendicular to flow that the predicted concentrations came into agreement with the observed ones. The resulting values of transverse dispersivity relative to longitudinal dispersivity were entirely inconsistent with the widelyaccepted notion that longitudinal dispersivities are almost always significantly greater than transverse dispersivities for plumes migrating under natural flow conditions. Figure A-1 shows the best-fitting values of transverse and longitudinal dispersivity from the 3DADE-PEST method as a function of distance from the observation well to the plume centerline (as well as the bestfitting groundwater velocities and sum of squares residuals between model and data as a function of this distance). There is clearly a sharp transition between reasonable and unreasonable relative values of the the longitudinal and transverse dispersivities that occurs at about a $2-\mathrm{m}$ distance between the observation well and the plume centerline. In the spreadsheet method, all reasonable matches to the PFBA breakthrough curve were obtained only if it was assumed that the flow direction was oriented at a 10-degree angle or more from the line connecting the injection and observation wells.

The second observation made when applying the two methods was that the best match to the PFBA breakthrough curve was highly nonunique. This nonuniqueness is apparent from the leveling-off of the sum of squares residuals in the 3DADE-PEST method as a function of distance between the observation well and the plume centerline for distances greater than about 3-4 $\mathrm{m}$ in Figure A-1. Note that all 3DADE-PEST calculations assumed a flow porosity of 0.2. Figure A-2 shows families of curves of flow velocity, transverse dispersivity, and longitudinal dispersivity as a function of the assumed angle between the flow direction and the line connecting the injection and observation wells for different assumed flow porosities. The 
longitudinal and transverse distances to $22 \mathrm{~S}$ from 22PA relative to the plume centerline are also plotted as a function of this angle in Figure A-2. Importantly, every point on every line in Figure A-2 has exactly the same sum of squares residuals between the model and data (i.e., the parameters associated with every point yield exactly the same fit). Points associated with observation well distances of $4 \mathrm{~m}$ and $8 \mathrm{~m}$ from the plume centerline (angles of approximately 15 degrees and 30 degrees between the line connecting the wells and the plume centerline, respectively) in Figure A-1 are shown on Figure A-2 to provide some perspective on the agreement between the parameter estimates provided by the two methods, which was generally quite good. Points associated with application of the spreadsheet method using the flow velocities or angles obtained from the 3DADE-PEST optimizations at the 4-m and 8-m transverse distances are shown in Figure A-2 for comparison. The differences between the methods can be attributed to the differences in the assumed initial distribution of tracer mass about the injection well and the fact that 3DADE does not simulate upgradient dispersion of tracer mass from the rectangular source block whereas the spreadsheet calculation allows such upgradient dispersion at the source. It should be noted that the plume translation calculations in both methods do not include a dependence on flow porosity, so the differences in the estimates from either method as a function of flow porosity (Figure A-2) are entirely attributable to the differences in the initial distribution of tracer mass near the injection well resulting from the differences in assumed porosity.

PEST provides estimation of parameter linear confidence intervals in addition to best estimates of the adjustable parameters in a model. The $95 \%$ confidence intervals for groundwater velocity, longitudinal dispersivity and transverse dispersivity for the cases with 4and 8-m distances (15- and 30-degree angles, respectively) between the observation well and the 
plume centerline are provided in Table A-1. The sensitivity of this model is probably non-linear and the linear confidence limits are quantitatively questionable, but they can provide a general indication of uncertainty. Note that if the transverse distances are not fixed, the parameter confidence intervals will be much larger because of the nonuniqueness that results when all parameters are allowed to vary simultaneously (discussed above).

Although a very wide range of flow velocities and flow directions yield acceptable matches to the PFBA breakthrough curve, other lines of evidence suggest that the flow direction should be at the smaller end of the range of angles relative to a straight line connecting wells 22PA and 22S. Specifically, the observation of an increased concentration of tracers from 22PA and a decreased concentration of tracer from 22PC immediately after the 159-day flow interruption in the forced-gradient cross-hole tracer test suggest an angle that is less than 45-degrees from the line connecting the wells. The rationale is that if the angle were greater than 45 -degrees, the tracer concentrations from 22PA would be expected to be initially lower when pumping resumed rather than higher, as observed. As Figure A-2 shows, smaller angles correspond to larger flow velocities using either the 3DADE-PEST or Spreadsheet methods of estimation. 


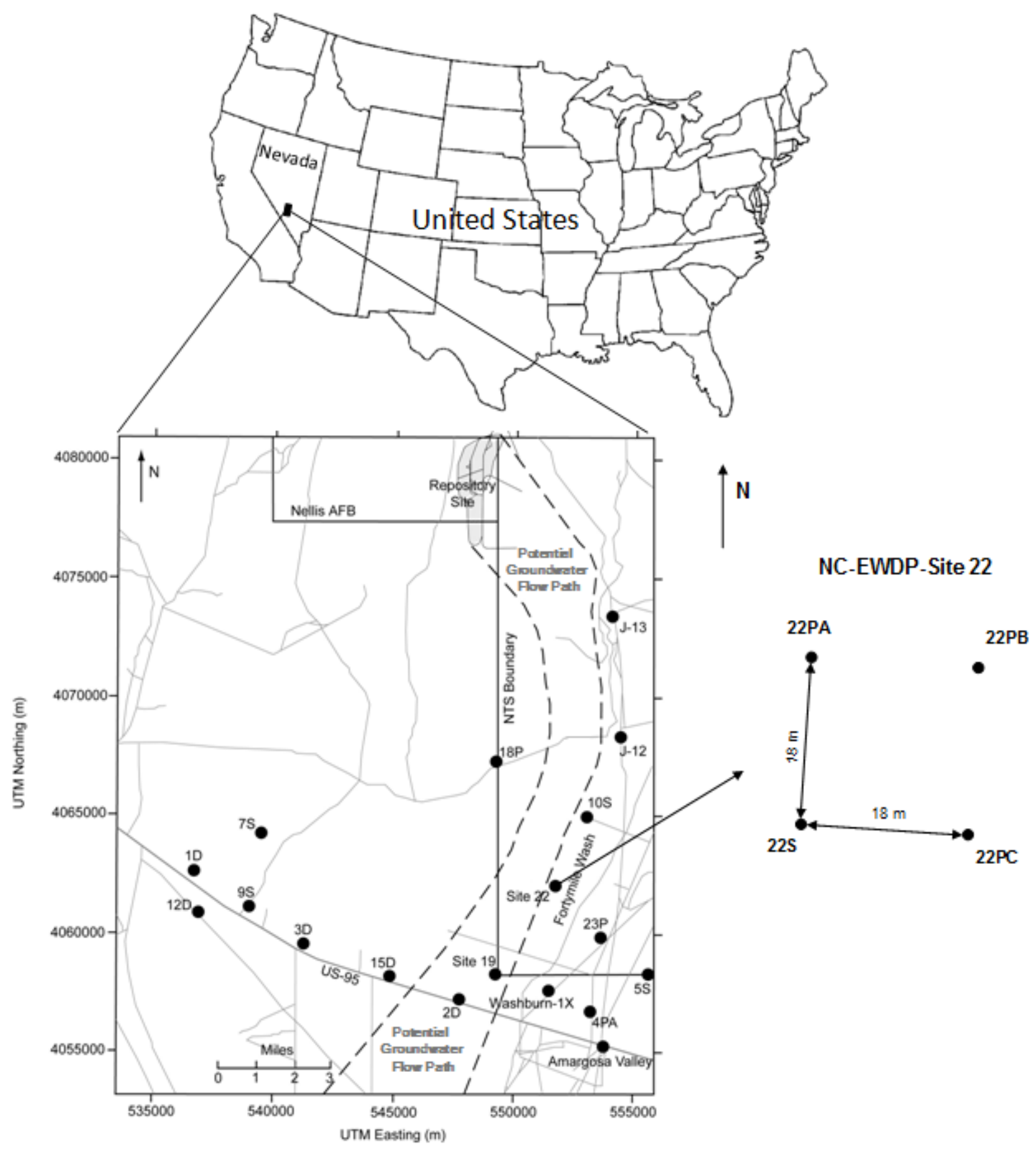

Figure 1. Location and layout of Nye County Early Warning drilling program Site 22 relative to Yucca Mountain repository site and projected groundwater flow path from Yucca Mountain (approximately bounded by the dashed lines). Note that the distances between wells are at the surface. The distance between 22PA and 22S in the tracer test interval is $16.7 \mathrm{~m}$ because of deviations of the borehole from vertical and the orientation at depth is almost due north-south. 


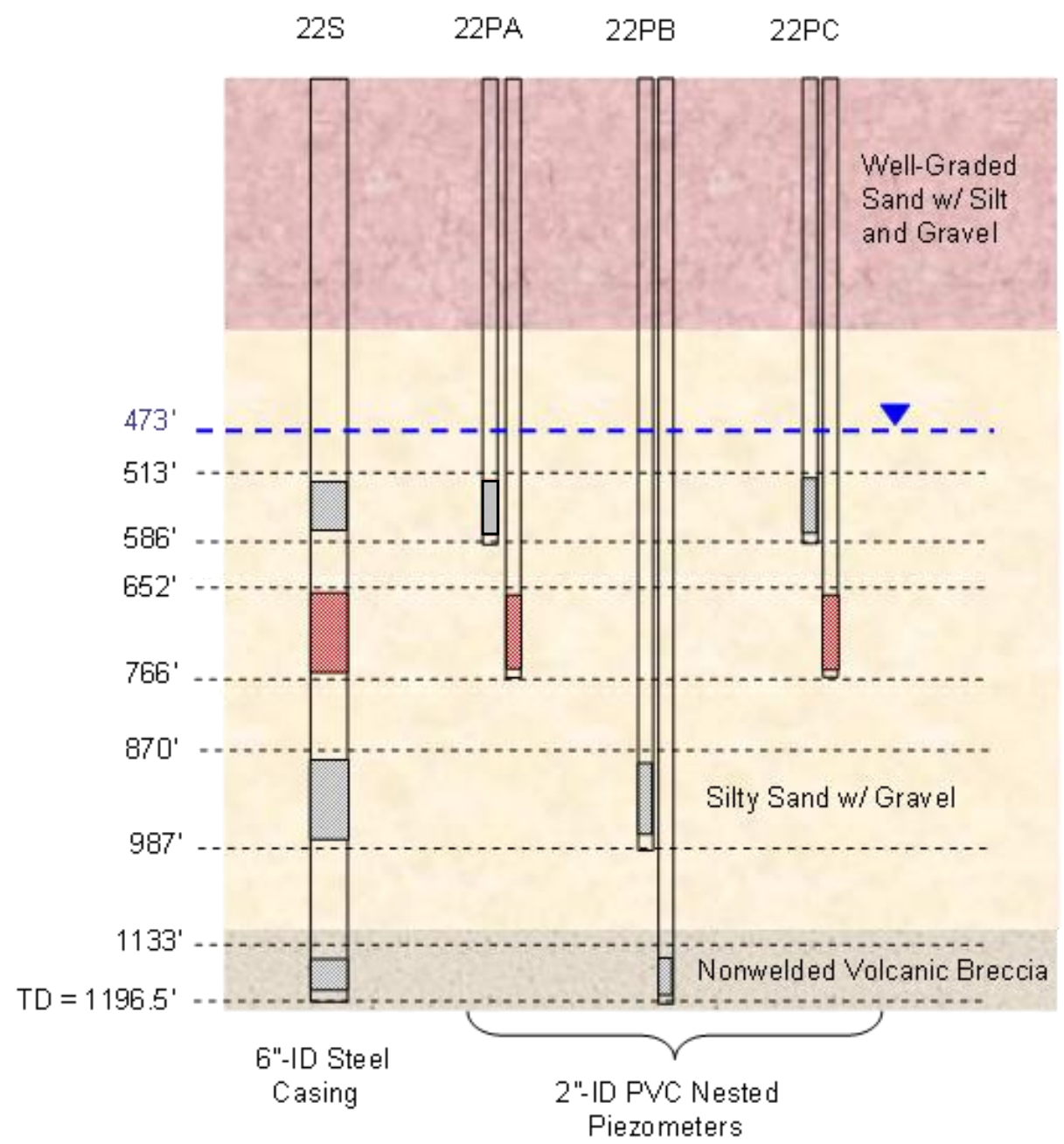

Figure 2. Simplified schematic diagram of Site 22 lithology and well completions. Red rectangles within wells indicate the wellscreens into which tracers were injected or withdrawn in the tracer tests (grey rectangles are other wellscreens). Dashed lines are approximate depths (below surface) of filter packs. Blue dashed line is the water table. The filter pack diameters for 22PA and 22PC in the test interval varied from $0.18 \mathrm{~m}$ in the upper part of the interval to $0.14 \mathrm{~m}$ in the lower part, and the filter pack for $22 \mathrm{~S}$ was $0.375 \mathrm{~m}$ in diameter. All intervals were determined to be isolated from each other outside the boreholes. 


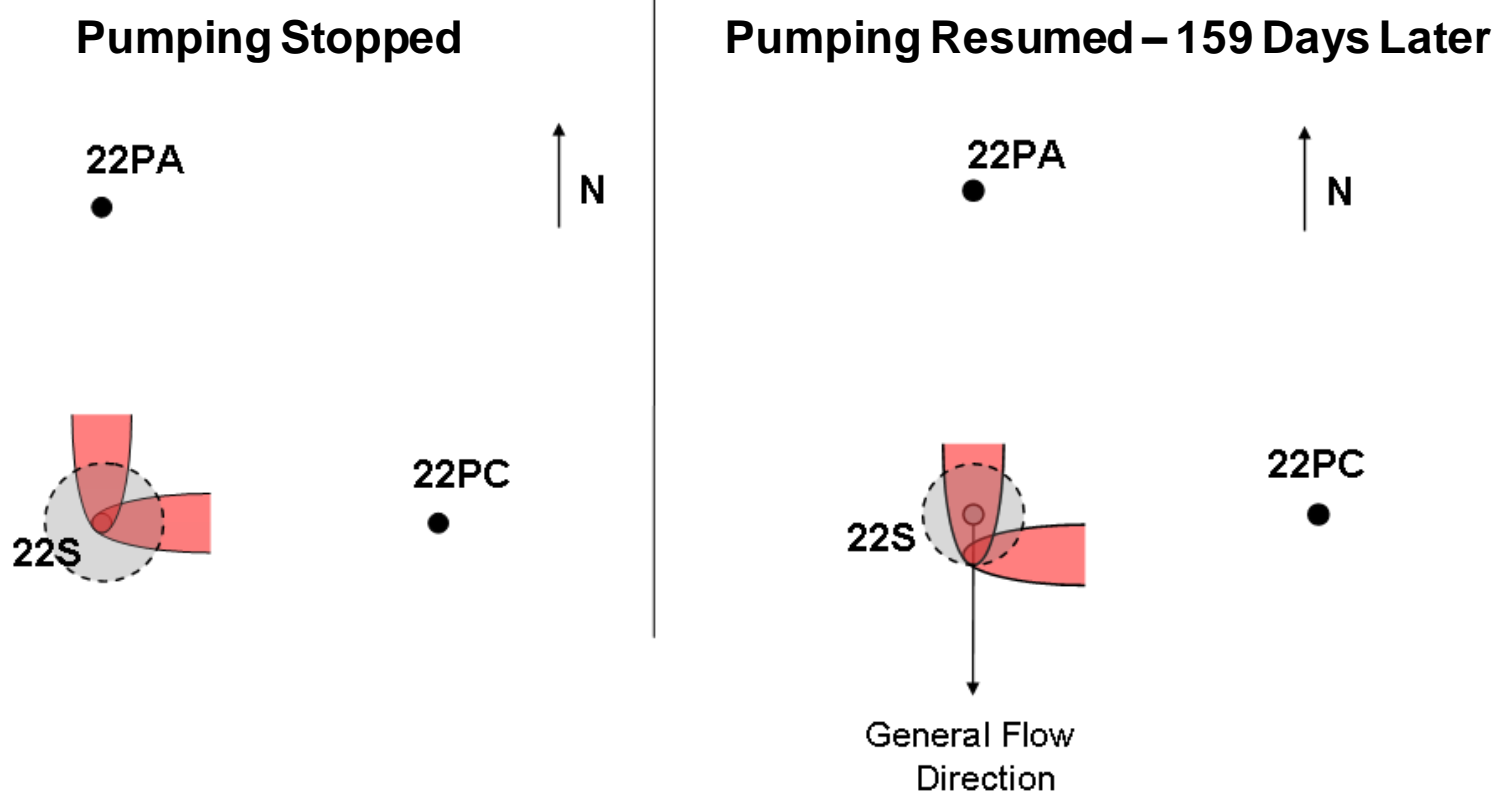

Figure 3. Schematic depictions of tracer plumes from 22PA and $22 \mathrm{PC}$ in the vicinity of $22 \mathrm{~S}$ before and after the flow interruption in the forced-gradient cross-hole tracer test. Grey circles represent the volume/area of aquifer produced in the first few hours after resuming pumping. 


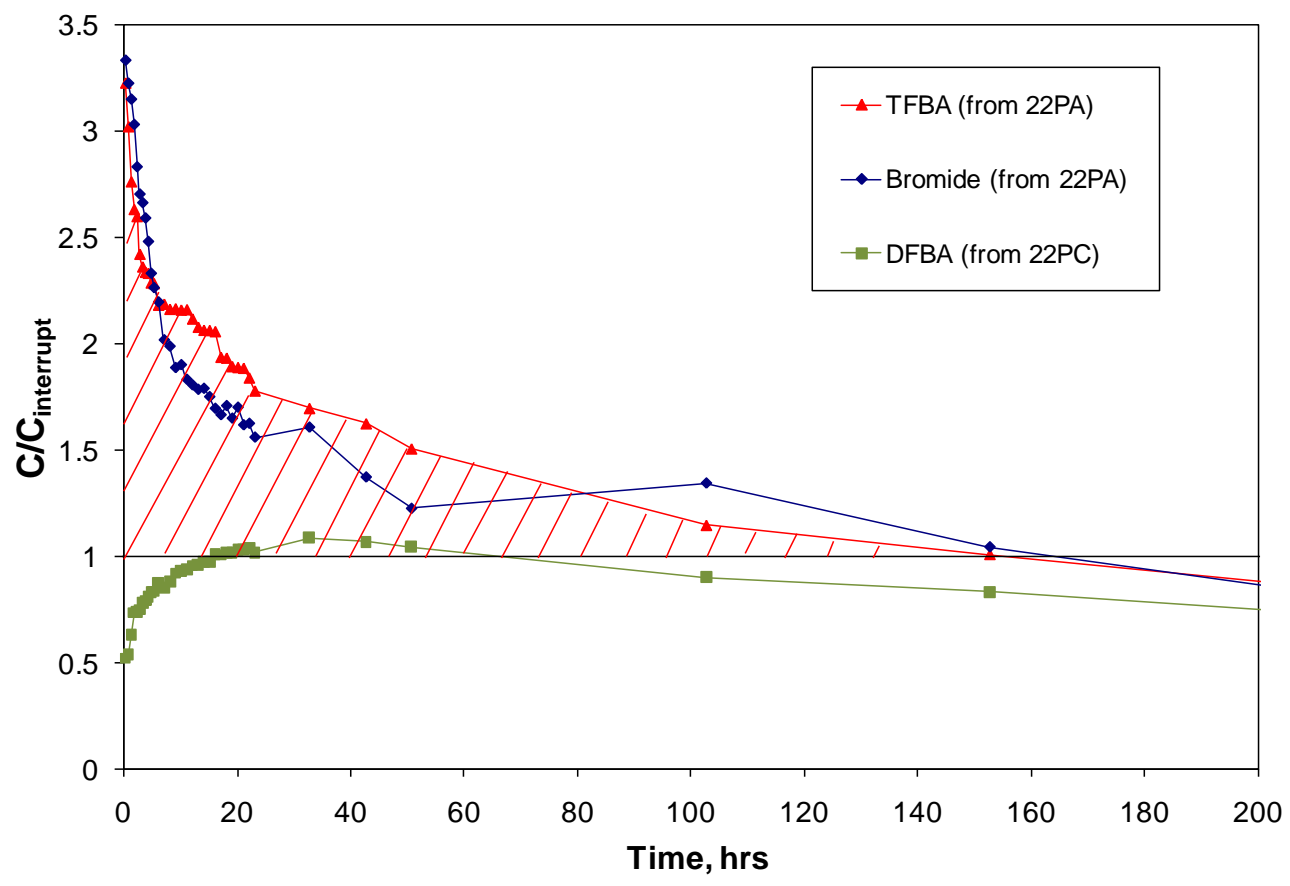

Figure 4. Tracer concentrations in well $22 \mathrm{~S}$ divided by tracer concentrations at time of flow interruption (159 days earlier) as a function of time since pumping resumed. Red hatched area represents the additional mass of TFBA recovered after pumping resumed. 


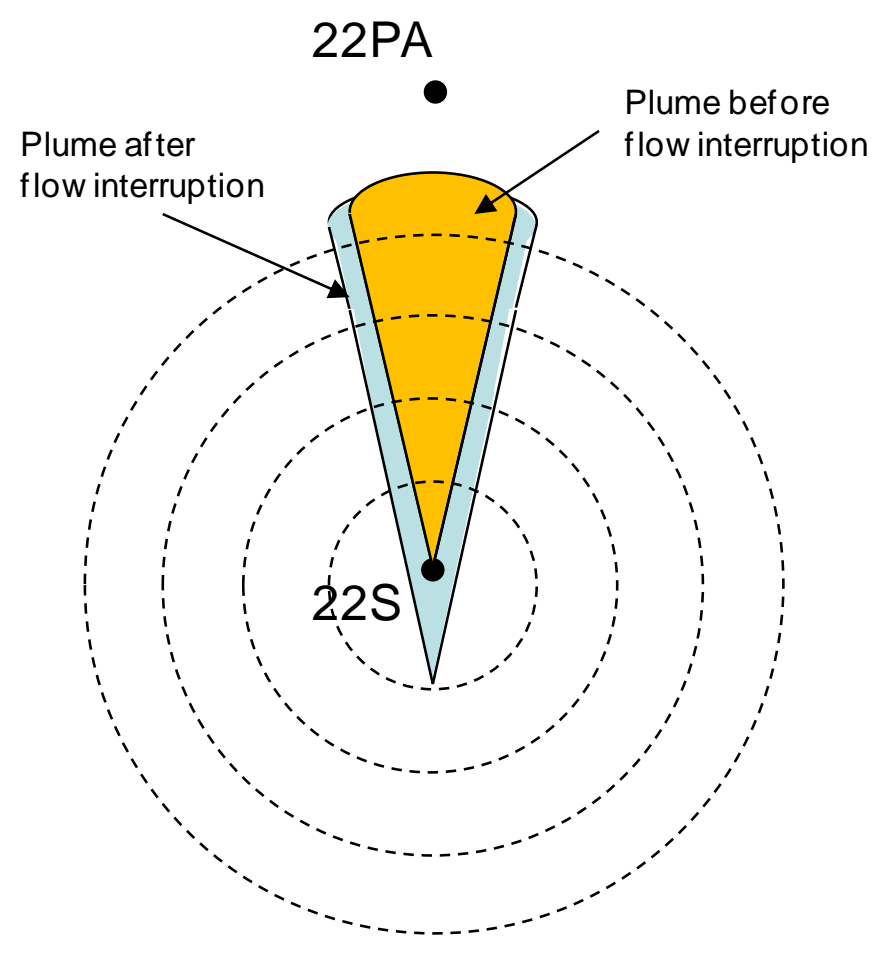

Figure 5. Depiction of wedge-shaped plume before and after flow interruption in a homogeneous, isotropic medium, with concentric rings corresponding to different aquifer volumes produced at times proportional to the radius of the rings squared. Between any two rings, the additional mass of tracer produced relative to what would have been produced without the flow interruption is represented by the blue area. The average tracer concentration produced between any two rings relative to the concentration that would have been produced is equal to the blue area plus the orange area divided by the orange area. 


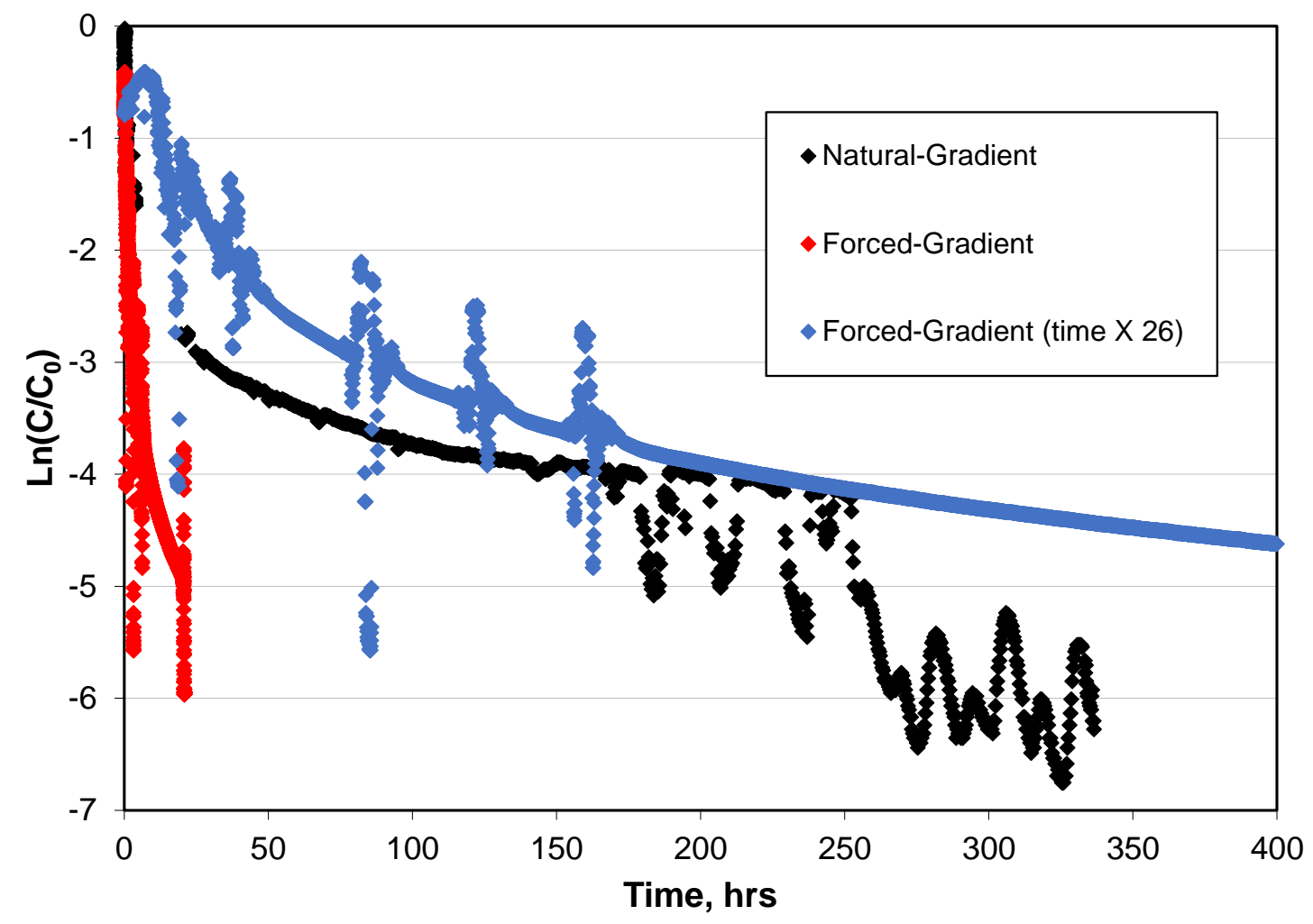

Figure 6. Log normalized tracer concentration histories in well 22PA at the beginning of the natural-gradient and forced gradient tracer tests. Blue curve is the same as the red curve with time scale multiplied by 26 . 


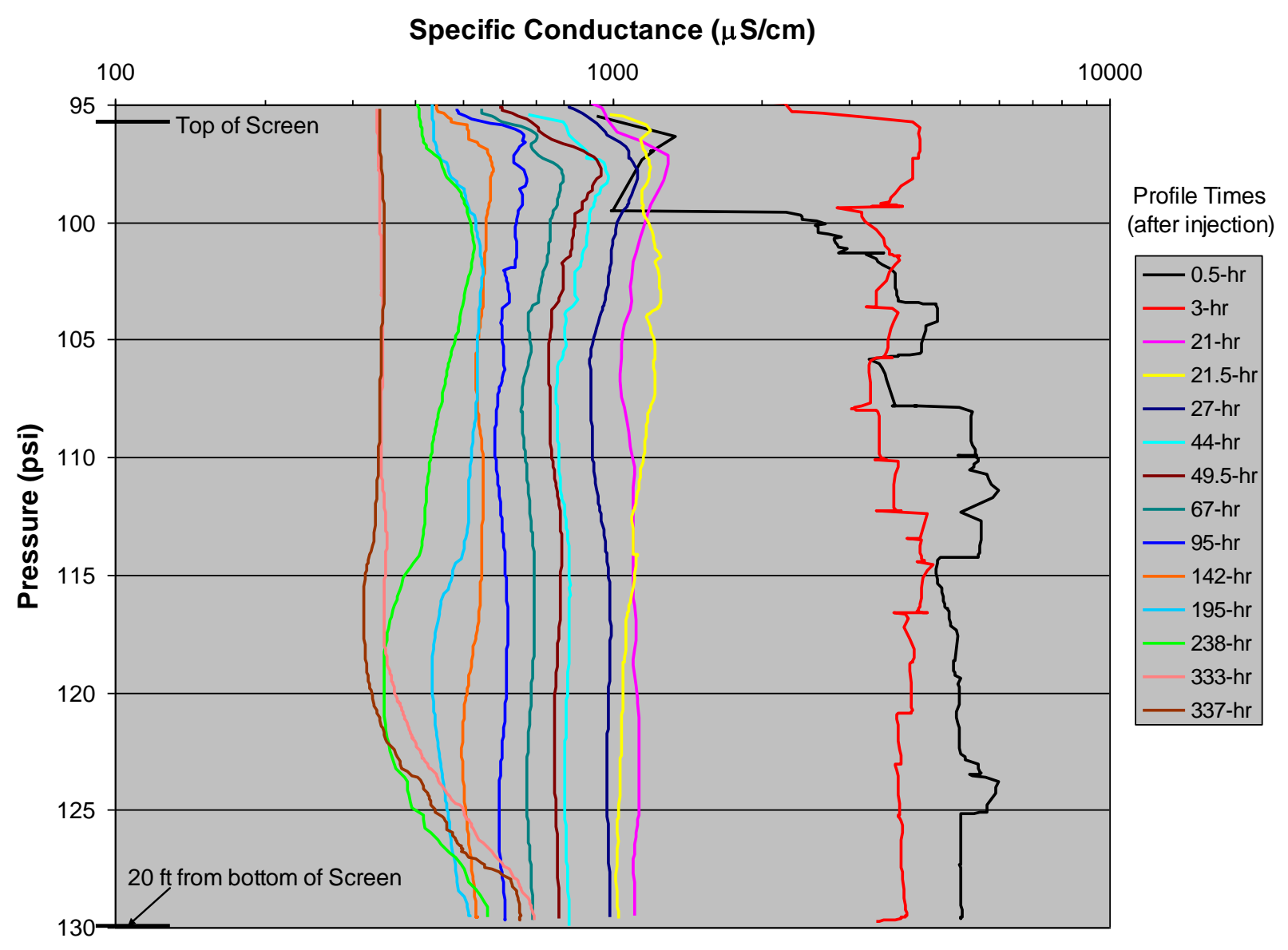

Figure 7. Specific conductance profiles in well 22PA (injection well) as determined with a downhole probe that was trolled up and down the injection interval after tracer injection in the natural gradient tracer test. Specific conductance is a proxy for tracer concentrations. 


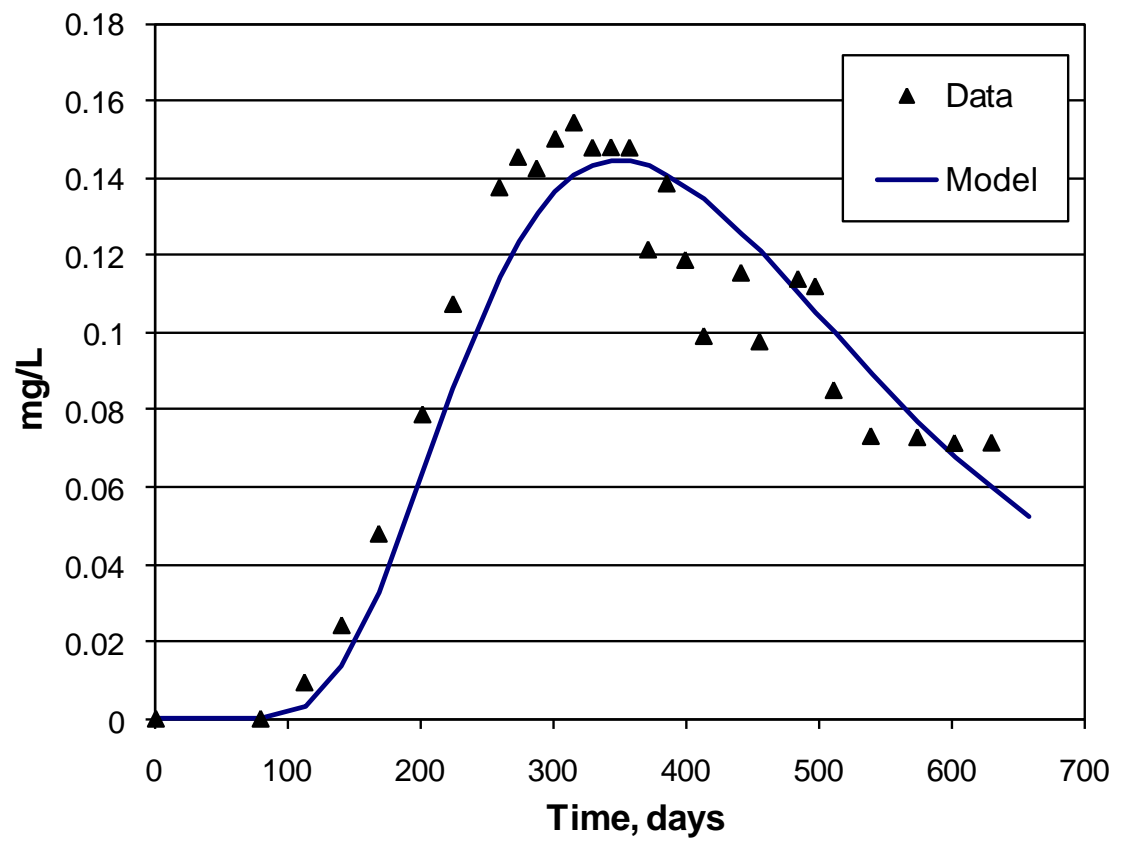

Figure 8. PFBA breakthrough curve in well $22 \mathrm{~S}$ in natural gradient tracer test and the best model fit to the data. 


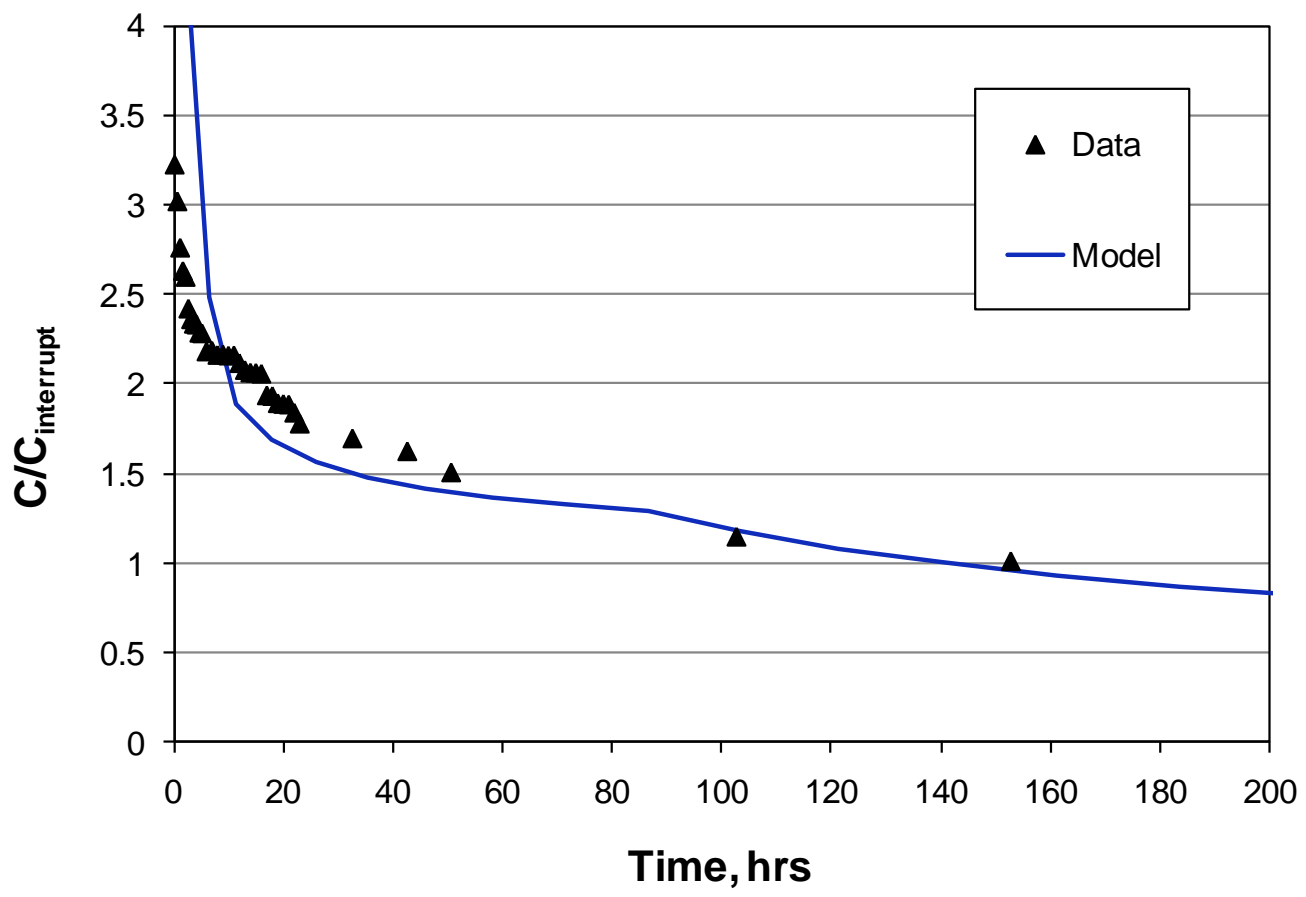

Figure 9. Simulated and observed normalized TFBA concentrations in well $22 \mathrm{~S}$ after resumption of pumping. Concentrations are normalized by dividing by the pre-flowinterruption concentration. The area under the two curves is the same. The area above $\mathrm{C} / \mathrm{C}_{\text {interrupt }}=1$ is 0.35 times the area below $\mathrm{C} / \mathrm{C}_{\text {interrupt }}=1$. 
Table 1. Ambient flow velocity estimates from multiple single-well tracer test method (m/year).

\begin{tabular}{cccc}
\hline Flow Porosity & $0.076^{\mathrm{a}}$ & $0.118^{\mathrm{a}}$ & $0.1^{\mathrm{b}}$ \\
\hline Peak Analysis $^{\mathrm{c}}$ & 7.2 & 5.8 & 6.4 \\
Mean Analysis $^{\mathrm{c}}$ & 12.4 & 10.0 & 11.0 \\
Tail Analysis $^{\mathrm{c}}$ & 32.9 & 26.3 & 29.3 \\
\hline
\end{tabular}

${ }^{a}$ Flow porosity estimates from the two forced-gradient tracer tests conducted between 22PA and 22S.

${ }^{\mathrm{b}}$ Approximate average of the two flow porosity estimates from forced-gradient tracer testing.

${ }^{c}$ See Reimus et al. (2003a) for complete description of the three methods that use the peak, mean and late (tail) arrival times to estimate flow velocities. 
Table 2. Ambient flow velocity estimates from the different methods assuming a flow porosity of 0.1 for the multiple single-well test method. Numbers in bold indicate the values considered to be the best estimates of the average velocity in the test interval from each method.

Method

Multiple Single-Well Tests (Peak/Mean/Tail Arrival Times)

Cross-Hole Flow Interruption (Wedge/Finger Plume)*

Multiple Tracer Decay Curve (Radial/Linear Flow)*

Natural Gradient (Simple Peak/Mean Arrival Time)*
Flow Velocity (m/yr)

$6.4 / 11 / 29.3$

$7.5 / 12.5$

$13.1 / 26.2$

$18.5 / 13$

${ }^{*}$ Indicates a method that provides an estimate that is independent of flow porosity. 
Table 3. Ambient flow velocity estimates at Site 22 using different natural gradient estimates and assuming a hydraulic conductivity of $12 \mathrm{~m}$ /day from cross-hole hydraulic testing in the tracer test interval (flow porosity assumed to be 0.1).

\begin{tabular}{|c|c|}
\hline Gradient Description & Flow Velocity $(\mathrm{m} / \mathrm{yr})$ \\
\hline Gradient between 22S and $191 \mathrm{M} 2^{\mathrm{a}}(0.00279 \mathrm{~m} / \mathrm{m})$ & 122 \\
\hline Gradient between $10 \mathrm{~S}$ and $19 \mathrm{IM}^{\mathrm{a}}(0.00196 \mathrm{~m} / \mathrm{m})$ & 86 \\
\hline Gradient between $10 \mathrm{~S}$ and $22 \mathrm{~S}(0.00069 \mathrm{~m} / \mathrm{m})$ & 30.5 \\
\hline
\end{tabular}

